\title{
Modelagem da vida de fadiga e do acúmulo de deformações permanentes em pavimentos asfálticos por meio de um modelo de dano contínuo
}

\author{
Victor Ferreira Teixeira1; Flávio Vasconcelos de Sousa²; Jorge Barbosa Soares ${ }^{3}$
}

\begin{abstract}
Resumo: É sabido que as condições das rodovias brasileiras têm grande impacto sobre a economia nacional. Diante disso, nasce a necessidade de uma busca por métodos de análise de pavimentos que possam prever com maior precisão as falhas as quais o pavimento está submetido. Dentre os principais métodos utilizados atualmente, pode-se citar os métodos puramente empíricos e aqueles mecanístico-empíricos que fazem uso da resposta estrutural do pavimento. A aprimoração de técnicas numéricas como o Método dos Elementos Finitos (MEF) ou o Método dos Elementos de Contorno (MEC) tem feito com que os métodos mecanístico-empíricos ganhem cada vez mais espaço e sejam mais usados no dimensionamento de pavimentos. O presente trabalho apresenta um modelo mecanístico que considera a evolução do dano causado pelos carregamentos e, conseqüentemente, possibilita uma melhor simulação da vida de fadiga da camada asfáltica. O modelo também possibilita uma melhor modelagem do acúmulo de deformação permanente nos pavimentos através do uso da Teoria da Viscoelasticidade, permitindo assim avaliar as duas principais falhas dos pavimentos flexíveis.
\end{abstract}

\begin{abstract}
It is known that the conditions of the Brazilian roadways have an enormous impact over the national economy. In face of this problem, there is a need for new methodologies for pavement analyses and design that can more accurately predict pavement distresses. Among the main methods currently used, one can find purely empirical methods and mechanistic-empirical methods which make use of the pavement structure response. The evolution of numerical methods such as the Finite Element Method (FEM) or the Boundary Element Method (BEM) has made the mechanistic-empirical methods more popular for pavement analysis applications. This work shows a mechanistic model that can consider the damage evolution caused by the loads and, subsequently, it can better predict the fatigue life of the asphalt layer. The approach also allows better modeling of permanent deformation in pavements, and therefore it is able to better predict the two most commons distresses in asphaltic pavements.
\end{abstract}

\section{INTRODUÇÃO}

As más condições das rodovias brasileiras têm grande impacto na economia nacional. A matriz de transportes do Brasil mostra que cerca de $60 \%$ do transporte de mercadorias é feito através do modal rodoviário. A importância das rodovias nas relações econômicas entre as diversas regiões brasileiras justifica a busca por métodos que possam prever com maior precisão as respostas às solicitações de tráfego às quais os pavimentos serão submetidos.

Dada a complexidade do comportamento dos materiais de pavimentação, é de fundamental importância destacar o uso de modelos computacionais que podem prever com maior precisão a vida útil dos pavimentos asfálticos. Dentre os modelos mais utilizados, destacam-se (i) os modelos micromecânicos, que podem levar em consideração fenômenos que acontecem na microescala e (ii) os modelos de dano contínuo, que,

\footnotetext{
${ }^{1}$ Victor Ferreira Teixeira, Department of Engineering Mechanics. University of Nebraska-Lincoln. Lincoln, USA (e-mail: teixeira@bigred.unl.edu).

${ }^{2}$ Flávio Vasconcelos de Sousa, Department of Engineering Mechanics. University of Nebraska-Lincoln. Lincoln, USA (e-mail: flaviovs@gmail.com)

${ }^{3}$ Jorge Barbosa Soares, Laboratório de Mecânica dos Pavimentos. Universidade Federal do Ceará. Fortaleza, CE, Brasil (e-mail: jsoares@det.ufc.br).
}

Manuscrito recebido em 2/8/2007 e aprovado para publicação em 19/10/2007. Este artigo é parte de TRANSPORTES, volume XV, número 2, de dezembro de 2007. ISSN: 1415-7713. baseados nas chamadas variáveis internas de estado, são capazes de modelar fenômenos da microescala de uma forma computacionalmente mais rápida, porém menos detalhada.

Os modelos micromecânicos, são aqueles que podem considerar a heterogeneidade dos materiais assim como a formação e propagação de trincas discretas. A maneira mais comumente usada na modelagem de trincas em pavimentos é através dos modelos de zona coesiva (Allen e Searcy, 2001). Uma das principais vantagens dos modelos micromecânicos é que estes permitem a realização de análises para diferentes geometrias da microescala, com diferentes frações volumétricas, sem requerer a realização de ensaios experimentais para determinação das propriedades constitutivas globais do material (Kim et al., 2006; Freitas, 2007). Os modelos multi-escala, os quais podem ser considerados modelos micromecânicos, são capazes de simular estruturas em aplicações reais conservando as características dos modelos micromecânicos.

Os modelos multi-escala têm, ainda, a vantagem de permitir a modelagem detalhada de fenômenos físicos que acontecem na microescala (Souza, 2005). A maior precisão é sempre desejada, mas a capacidade computacional atual não permite a realização de simulações multi-escala muito detalhadas, como as requeridas na análise de pavimentos.

Por outro lado, os modelos de dano contínuo representam mudanças na microescala dos materiais de 
uma maneira homogeneizada sem requerer a análise da microescala. Essas mudanças (pequenas trincas, mudanças químicas, entre outros) são representadas por variáveis internas de estado, cuja evolução deve ser determinada experimentalmente para cada mistura, sendo esta sua principal desvantagem. Os principais modelos de dano contínuo para materiais viscoelásticos são baseados nos estudos de Schapery (1990a, 1990b), Park et al. (1996) e Lee e Kim (1998a, 1998b). Estes modelos definem a lei de evolução das variáveis internas de estado com base na função de energia de deformação e utilizam o princípio da correspondência viscoelástica (Schapery, 1984) para considerar o comportamento dependente do tempo desses materiais.

Uma vez que trincas discretas não são explicitamente modeladas, o esforço computacional nos modelos de dano contínuo é reduzido drasticamente quando comparado ao dos modelos micromecânicos. Com isso, o uso de modelos de dano contínuo torna-se mais viável atualmente para a modelagem de pavimentos, a qual exige a simulação de um grande número de ciclos de carregamento.

O presente estudo tem como principal objetivo o uso de um modelo de dano contínuo capaz de considerar a vida de fadiga e as deformações permanentes, que são os principais fatores de deterioração em pavimentos asfálticos. Modelos como o apresentado neste trabalho representam uma contribuição para o contínuo avanço da Mecânica dos Pavimentos, que busca a compreensão do pavimento como um sistema de camadas no qual devem estar compatibilizadas as tensões e deslocamentos solicitantes com as propriedades dos materiais e espessuras das camadas (Medina e Motta, 2005).

\section{PROBLEMA DE VALOR DE CONTORNO INICIAL (PVCI)}

Considere um corpo qualquer de volume $V$ e superfície $\partial V$, dividida em duas partes: $\partial V_{t}$, onde as trações são conhecidas, e $\partial V_{u}$, onde os deslocamentos são conhecidos (Figura 1). As principais variáveis utilizadas nesse modelo de dano contínuo são o vetor dos deslocamentos $u_{i}\left(x_{i}, t\right)$, o tensor de tensões de Cauchy $\sigma_{i j}\left(x_{i}, t\right)$, o tensor das deformações $\varepsilon_{i j}\left(x_{i}, t\right)$ e a variável interna de estado $\alpha\left(x_{i}, t\right)$, onde $t$ (tempo) e $x_{i}$ (coordenadas) são as variáveis independentes. Note-se que a notação indicial de Einstein fora utilizada na representação das variáveis.

Através do balanço das forças no corpo, a segunda lei de Newton pode ser escrita da seguinte forma:

$$
\sigma_{i j},{ }_{j}+\rho f_{i}=\rho \frac{D v_{i}}{D t}
$$

Onde a vírgula (,) denota derivada, $D / D t$ é a derivada tomada para uma partícula de material, $\rho$ é a densidade do material, $f$ representa as forças de corpo (peso próprio) por unidade de massa e $v$ a velocidade. Desconsiderando os efeitos do peso próprio e considerando condições quasi-estáticas (velocidade igual a zero), a mesma equação pode ser reescrita como:

$$
\sigma_{i j},_{j}=0 \text { em } V
$$

A equação de conservação de momento angular pode ser expressa na seguinte forma:

$$
\sigma_{i j}=\sigma_{j i} \text { em } V
$$

Assumindo-se que as deformações são infinitesimais, a relação entre as deformações e os deslocamentos é dada pela seguinte equação:

$$
\varepsilon_{i j}=\frac{1}{2}\left(u_{i, j}+u_{j, i}\right) \text { em } V
$$

Finalmente, a relação contitutiva para um material elástico linear é dada por:

$$
\sigma_{i j}\left(x_{k}, t\right)=\lambda \varepsilon_{k k}\left(x_{k}, t\right) \delta_{i j}+2 \mu \varepsilon_{i j}\left(x_{k}, t\right)
$$

Onde $\lambda$ e $\mu$ são as constantes de Lamé.

Para um material viscoelástico, que apresenta comportamento dependente não apenas das deformações, mas também da taxa de carregamento, a equação constitutiva pode ser expressa na seguinte integral de convolução (Christensen, 1982):

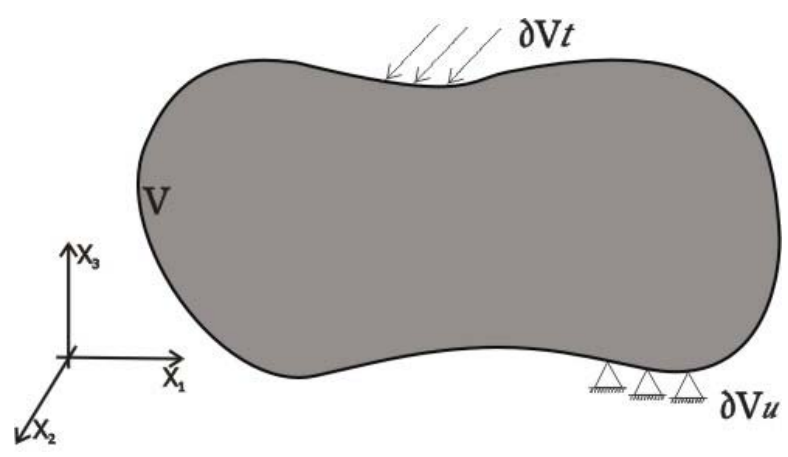

Figura 1. Corpo qualquer 


$$
\sigma_{i j}\left(x_{k}, t\right)=\int_{0}^{t} C_{i j k l}(t-\tau) \frac{\partial \varepsilon_{k l}\left(x_{k}, \tau\right)}{\partial \tau} d \tau
$$

Onde $C_{i j k l}(t)$ é o tensor dos módulos de relaxação, que é dependente do tempo e pode ser obtido através de ensaios laboratoriais, e $\tau$ é a variável de integração.

Para completar a definição do Problema de Valor de Contorno Inicial (PVCI), assume-se que os valores iniciais de todas as variáveis são conhecidos e iguais a zero.

$$
\begin{gathered}
\sigma_{i j}\left(x_{k}, t=0\right)=0 \\
\varepsilon_{i j}\left(x_{k}, t=0\right)=0 \\
u_{i}\left(x_{k}, t=0\right)=0
\end{gathered}
$$

Também é admitido que são conhecidas à priori as condições de contorno, quais sejam, as trações $t_{i}$, ou seja, tensões nas bordas (em inglês tractions), e os deslocamentos $u_{i}$ :

$$
\begin{gathered}
t_{i}=\hat{t}_{i} \text { em } \partial V_{t} \\
u_{i}=\hat{u}_{i} \text { em } \partial V_{u}
\end{gathered}
$$

Onde ^ $\wedge$ significa que a variável é conhecida.

\subsection{Modelo de dano contínuo}

No modelo de dano contínuo considerado, a equação constitutiva dos materiais viscoelásticos é reescrita da seguinte forma:

$$
\sigma_{i j}\left(x_{k}, t\right)=[1-\alpha(t)] \int_{0}^{t} C_{i j k l}(t-\tau) \frac{\partial \varepsilon_{k l}\left(x_{k}, \tau\right)}{\partial \tau} d \tau
$$

Onde $\alpha(t)$ é a variável interna de estado que representa o dano no material. A lei de evolução de $\alpha(t)$ usada no presente estudo é baseada na proposta feita por Allen e Searcy (2001) e é dada por:

$$
\begin{gathered}
\frac{\partial \alpha}{\partial t}=A[\lambda(t)]^{m}, \text { para } \frac{\partial \alpha}{\partial t}>0 \text { e } \alpha<1 \\
\frac{\partial \alpha}{\partial t}=0, \text { para } \frac{\partial \alpha}{\partial t} \leq 0 \text { ou } \alpha=1
\end{gathered}
$$

Onde $A$ e $m$ são constantes a serem determinadas experimentalmente e $\lambda(t)$ é definida como:

$$
\lambda(t) \equiv \sqrt{\varepsilon_{i j} \varepsilon_{i j}}
$$

A incrementalização do modelo acima de modo a implementá-lo num código de Elementos Finitos foi feita baseado nos trabalhos de Zocher (1995) e Seidel et al. (2004).

\section{SIMULAÇÃO DE PAVIMENTOS ASFÁLTICOS}

$\mathrm{Na}$ análise realizada neste estudo, foi considerada uma estrutura típica de um pavimento asfáltico constituída por 3 camadas sendo a primeira uma camada asfáltica viscoelástica, a segunda uma base elástica e a terceira um subleito elástico. As próximas seções tratam dos materiais utilizados, dos carregamentos considerados e da geometria usada no problema, respectivamente.

\subsection{Propriedades construtivas}

Neste trabalho foram admitidas que as subcamadas (base e subleito) apresentam comportamento linear elástico e seus módulos de elasticidade foram assumidos 300 e 100MPa e coeficientes de Poisson de 0,40 e 0,45 , respectivamente (Huang, 2004). No caso do revestimento asfáltico, este foi considerado viscoelástico linear (Souza e Soares, 2003), sendo os coeficientes da série de Prony $\left(E_{i}\right.$ e $\left.\rho_{i}\right)$ retirados de Gibson et al. (2002) e mostrados na Tabela 1.

\begin{tabular}{ccc} 
Tabela 1. Propriedades Viscoelásticas (Gibson et al., 2002) \\
\hline \multicolumn{3}{c}{ Módulo de Relaxação } \\
i & $E_{i}(\mathrm{~Pa})$ & $\rho_{i}$ (segundos) \\
\hline$E_{\infty}$ & 412,8 & - \\
1 & $1,43 \mathrm{E}+04$ & $1,50 \mathrm{E}+10$ \\
2 & $3,21 \mathrm{E}+04$ & $8,01 \mathrm{E}+08$ \\
3 & $7,42 \mathrm{E}+04$ & $4,27 \mathrm{E}+07$ \\
4 & $1,80 \mathrm{E}+05$ & $2,28 \mathrm{E}+06$ \\
5 & $4,59 \mathrm{E}+05$ & $1,22 \mathrm{E}+05$ \\
6 & $1,23 \mathrm{E}+06$ & $6,49 \mathrm{E}+03$ \\
7 & $2,96 \mathrm{E}+06$ & $3,47 \mathrm{E}+02$ \\
8 & $5,29 \mathrm{E}+06$ & $1,85 \mathrm{E}+01$ \\
9 & $6,53 \mathrm{E}+06$ & $9,87 \mathrm{E}-01$ \\
10 & $5,73 \mathrm{E}+06$ & $5,27 \mathrm{E}-02$ \\
11 & $3,85 \mathrm{E}+06$ & $2,81 \mathrm{E}-03$ \\
12 & $2,16 \mathrm{E}+06$ & $1,50 \mathrm{E}-04$ \\
\hline & &
\end{tabular}

\subsection{Carregamentos}

A passagem de veículos representa a principal carga a qual os pavimentos são submetidos. A distribuição desta carga tem sido foco de muitos estudos (Schapery e Tielking, 1977; Tielking, 1983). Tielking e Roberts (1987) mostraram que a pressão de contato entre pneu-pavimento, apesar de ser fortemente afetada pela pressão interna dos pneus, não é igual a mesma. Este estudo também mostrou que as cargas não são igualmente distríbuidas no pavimento. Estudos feitos pela Tekscan, Inc. mostram que a carga é distribuída de acordo com a Figura 2.

Entretanto, no presente trabalho a carga foi considerada uniformemente distribuída sobre uma área circular de $15 \mathrm{~cm}$ de raio. Foi admitido um carregamento 


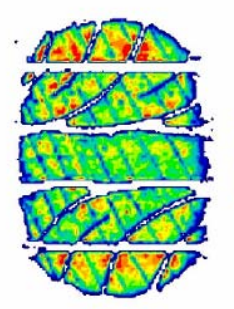

(a) Veículo de passageiros

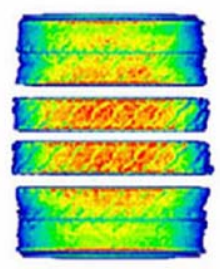

(b) Caminhão

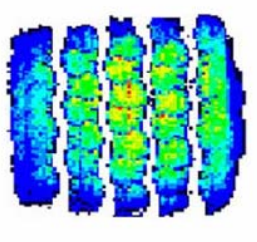

(c) Veículo fora de estrada

Figura 2. Distribuição da carga no pavimento (Tekscan, 2007)

de $45 \mathrm{kN}$, correspondente a uma pressão de $630 \mathrm{kPa}$.

Sabe-se que o pulso de carga pode ser aproximado por um carregamento semi-senoidal. No presente estudo, foi considerado um pulso de aplicação da carga de $0,1 \mathrm{~s}$ seguido de $0,9 \mathrm{~s}$ de repouso. Neste trabalho foram simulados apenas 200 ciclos de carga devido à limitações computacionais. A Figura 3 mostra como a pressão varia com o tempo em um ciclo de carga. Como as tensões são maiores durante a aplicação do pulso de carga, as taxas de deformação nesse período também são maiores, o que requer o uso de intervalos de tempo menores que possam captar melhor o comportamento dependente do tempo dos materiais.

Vale salientar que cargas térmicas, assim como outros fatores ambientais, não são consideradas por efeito de simplificação.

\subsection{Geometria do problema}

Como citado anteriormente, o problema consiste de uma estrutura de um pavimento com 3 camadas, sendo a primeira de concreto asfáltico com $10 \mathrm{~cm}$ de espessura, a segunda uma base de agregados de $20 \mathrm{~cm}$ e a terceira é um subleito infinito. Elementos infinitos, que são elementos com funções de forma especiais, também foram utilizados para se dimininuir o número de elementos distantes da região carregada e conse- qüentemente se reduzir o esforço computacional. A malha de elementos finitos foi gerada pelo programa MTOOL 4.0 (2001) e consiste de uma malha com 586 elementos quadrilaterais Q4 e 37 elementos infinitos também quadrilateriais Q4.

$\mathrm{O}$ uso dos elementos infinitos foi feito de acordo com recomendações dadas em NCHRP (2004). O estudo sugere o uso de distâncias verticais entre 60 e $190 \mathrm{~cm}$ entre a localização dos elementos infinitos e o ponto de aplicação da carga. Na malha utilizada para este trabalho, foi adimitida uma distância igual a $150 \mathrm{~cm}$. Para a distância horizontal, também foi usado $150 \mathrm{~cm}$ entre o centro do carregamento e os elementos infinitos. A distância vertical indicada no mesmo estudo é calculada baseando-se na solução de Boussinesq. A Figura 4 mostra a geometria do problema, as condições de contorno e a malha de elementos finitos utilizada, mostrando também os elementos infinitos.

\section{ANÁLISES E RESULTADOS}

Depois de definidos a geometria do problema, os carregamentos e os materiais, pode-se proceder à análise das tensões e deformações no pavimento. Foram realizadas análises para os casos com dano e sem dano. Dentre os casos nos quais se considerou o dano, foram

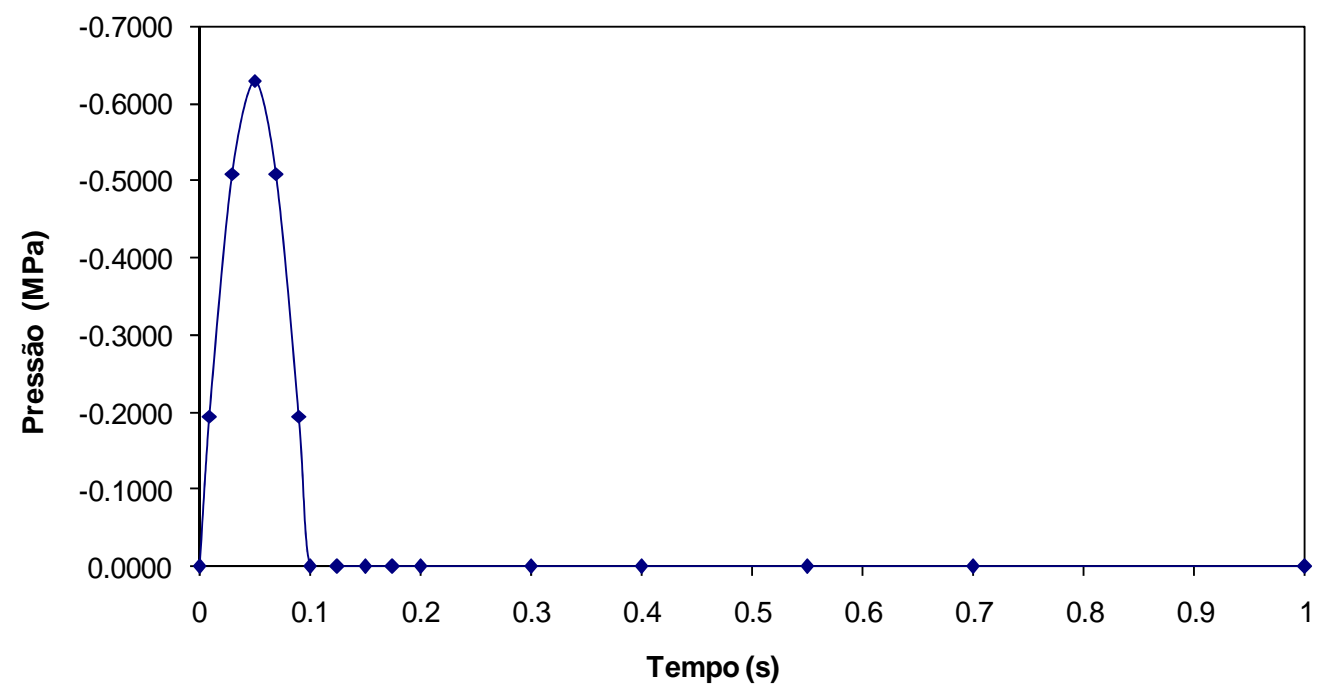

Figura 3. Ciclo de carga 


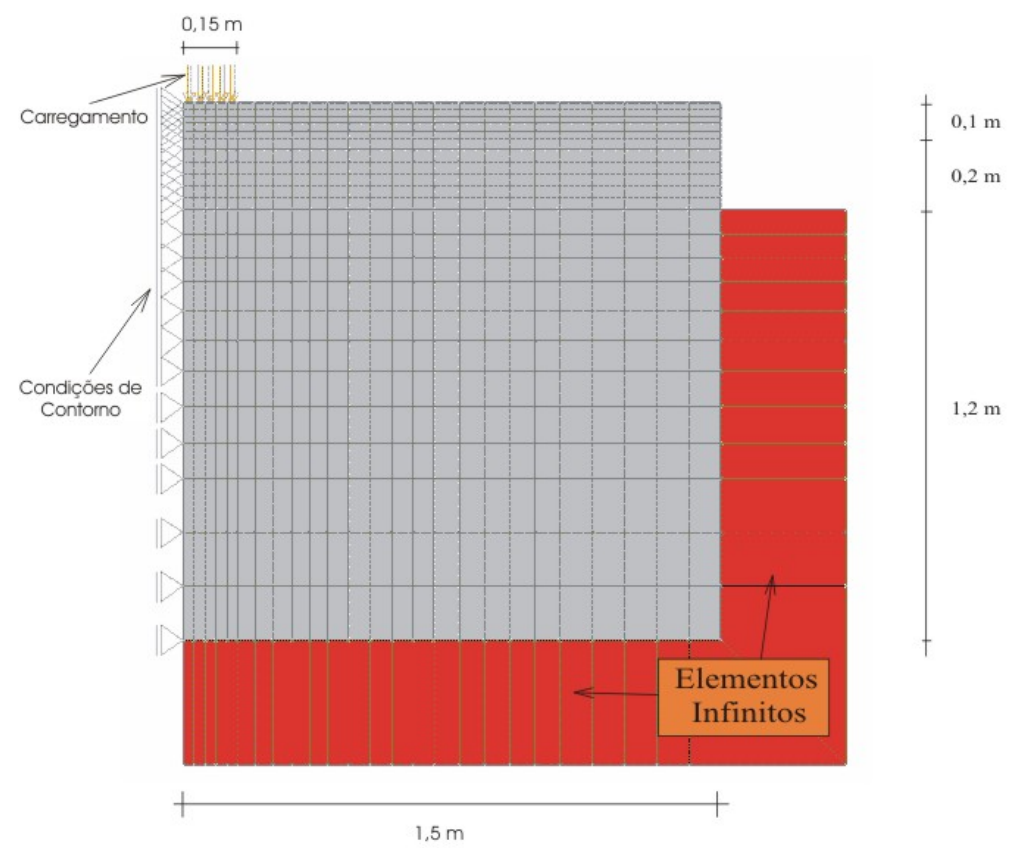

Figura 4. Malha de elementos finitos

testados vários valores para o parâmetro $A$ da Equação 10 para que se possa observar a influência do mesmo na resposta do modelo. Os valores utilizados são mostrados na Tabela 2.

Tabela 2. Parâmetros para simulação

\begin{tabular}{ccc}
\hline \multirow{2}{*}{ Análise } & \multicolumn{3}{c}{ Parâmetro } \\
\cline { 2 - 3 } & $\boldsymbol{A}$ & $\boldsymbol{m}$ \\
\hline 1 & 10 & 0,5 \\
2 & 5 & 0,5 \\
3 & 0,25 & 0,5 \\
4 & \multicolumn{2}{c}{ Sem Dano } \\
\hline
\end{tabular}

A Figura 5 compara a função de dano $\alpha(t)$ para três diferentes casos: caso $1(A=10$ e $m=0,5)$, caso 2 ( $A=5$ e $m=0,5)$ e caso $3(A=0,25$ e $m=0,5)$. A referida figura mostra o efeito do coeficiente $A$ na evolução do dano. Pode-se observar que o dano aumenta com o aumento de $A$, conforme esperado.

A Figura 6 mostra os resultados das deflexões máximas sofridas pelo pavimento comparando-se diferentes valores de $A$. Esta figura também apresenta o caso 4 (viscoelástico linear sem dano). A partir da referida figura também se pode observar que é possível simular o acúmulo de deformação permanente através da utilização do modelo proposto. Trabalhos realizados utilizando testes experimentais como o Hamburg ou o APA (Asphalt Pavement Analyzer) mostram tendências semelhantes às obtidas pelo presente modelo (Lutif, 2007).

A Figura 7 mostra o acúmulo do dano para diferentes ciclos de carga. Na Figura 7a, que representa 50 ciclos de carga, o dano é ainda pequeno (menor que 10\%). Já na Figura 7d, que representa 200 ciclos de

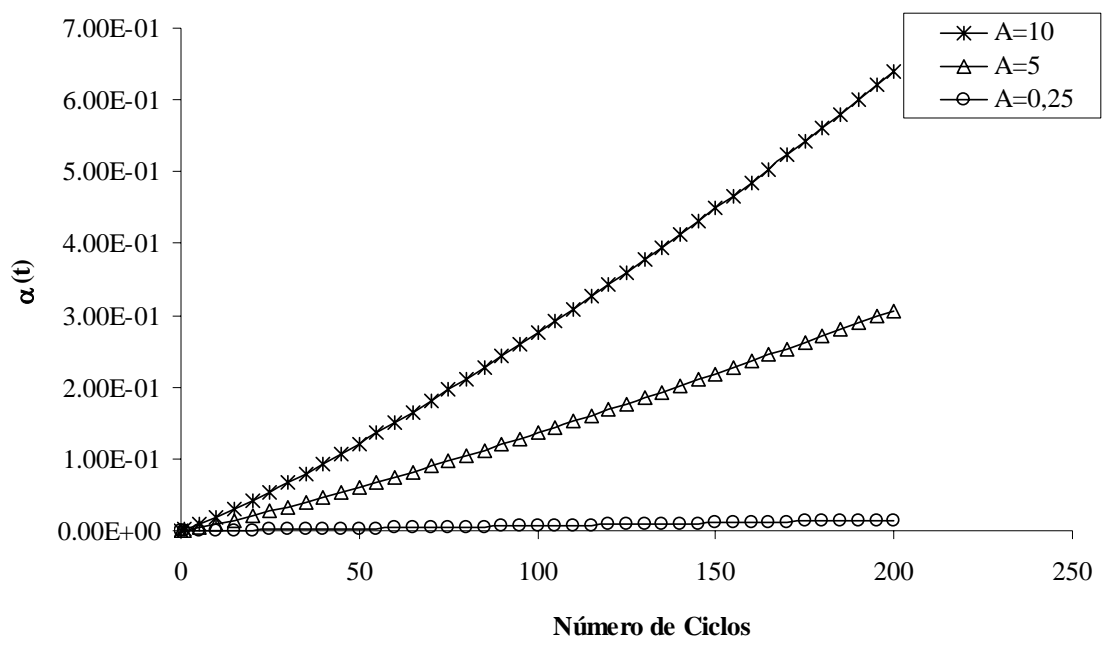

Figura 5. Efeito do parâmetro $A$ no crescimento do dano 


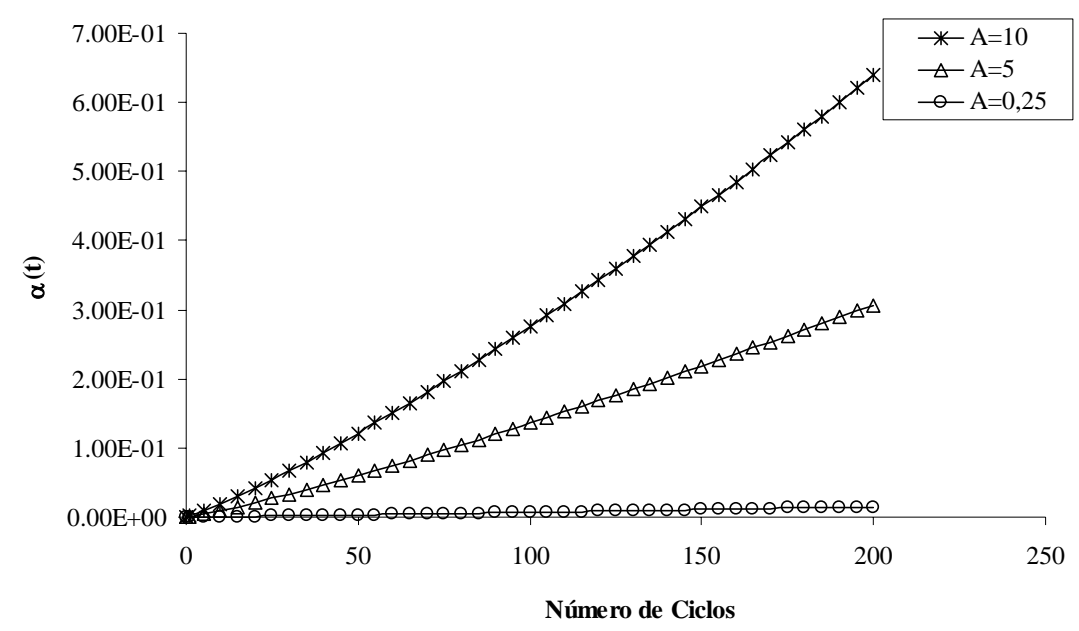

Figura 6. Deflexões máximas

carga, observa-se que o dano atinge valores maiores que $50 \%$. Vale salientar que o dano é acumulado principalmente na superfície e no fundo do revestimento, onde a magnitude das tensões é maior.

Dentre os principais mecanismos de falha dos pavimentos asfálticos, pode-se citar a deformação permanente e o aparecimento de trincas devido à fadiga dos materiais. As trincas devido à fadiga comumente se iniciam como microtrincas no fundo da camada de revestimento, onde as concentrações de trações são mais altas. Depois de repetidos ciclos de carga, essas microtrincas crescem e propagam-se para a superfície, causando a falha do pavimento. A deformação permanente, que geralmente ocorre na trilha de roda, está relacionada a diversos fatores como o uso de um ligante inadequado à temperatura de serviço, o excesso de ligante ou a indevida angularidade dos agregados. Entretanto, uma das principais causas deste problema é a deformação permanente do subleito ou da base (Huang, 2004).
Sabendo que os principais mecanismos de falha dos pavimentos são originados, principalmente, devido as grandes concentrações de tração no fundo da camada asfáltica e/ou à compressão no subleito ou na base, esses são importantes parâmetros que devem ser analisados. As Figuras 8, 9 e 10 mostram, respectivamente, as tensões horizontais no fundo da camada asfáltica $\left(\sigma \mathrm{xx}_{\text {revestimento }}\right)$, as tensões de compressão vertical no topo da base ( $\sigma \mathrm{yy}_{\text {base }}$ ) e as tensões verticais no topo do subleito ( $\left.\sigma \mathrm{yy}_{\text {subleito }}\right)$.

A partir da Figura 8 pode-se observar que à medida que o dano se acumula, as tensões no fundo da camada asfáltica diminuem. Esse comportamento se deve ao fato de que, com o aumento do dano, a rigidez da camada asfáltica diminui fazendo com que a mesma deforme mais e absorva menos as tensões. À medida que o revestimento vai perdendo a sua capacidade de absorção das tensões, as camadas inferiores, assumidas elásticas lineares sem dano no presente trabalho, são mais exigidas e absorvem maiores tensões con-

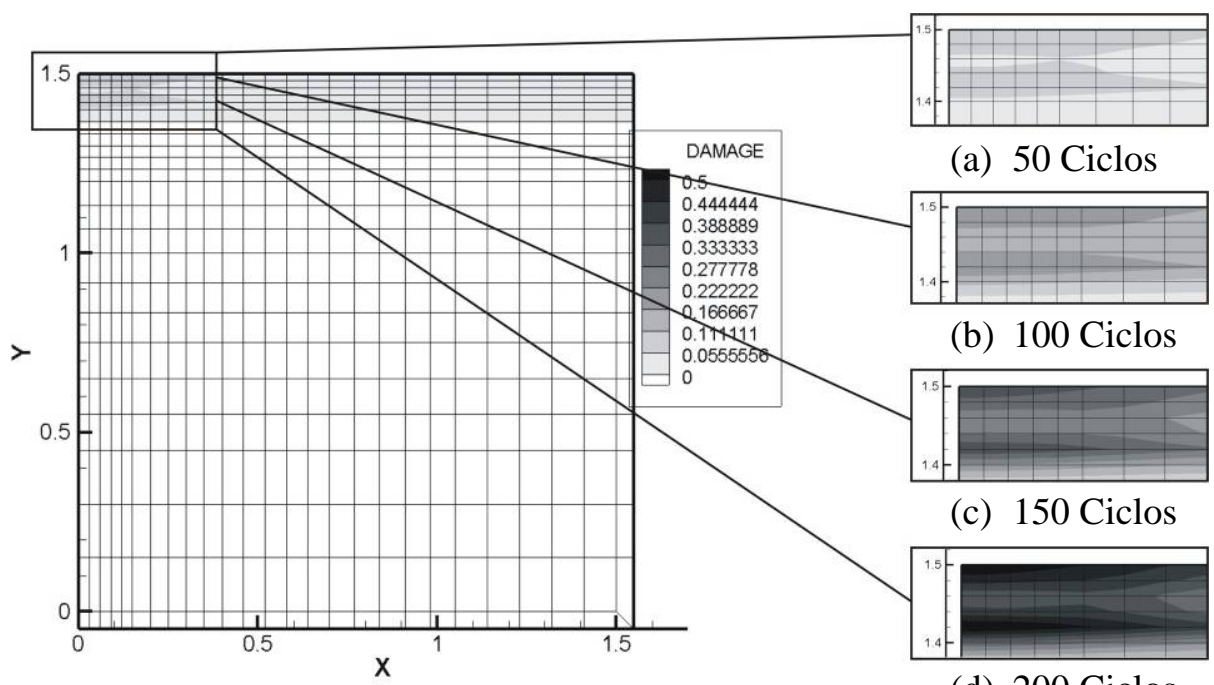

Figura 7. Dano para diferentes ciclos de carga 
forme mostrado nas Figuras 9 e 10, respectivamente, fáltica, que diminui à medida que o dano aumenta. para o topo da base e para o topo do subleito.

A Figura 11 mostra a redução das tensões horizontais no fundo da camada asfáltica com a progressão do número de ciclos de carga, especificamente para a interface entre o revestimento e a base. Estas tensões caem, afinal são proporcionais à rigidez da camada as-

\section{CONSIDERAÇÕES FINAIS}

O presente trabalho apresenta um modelo numérico para o cálculo de tensões e deformações em pavimentos asfálticos capaz de considerar a evolução do dano

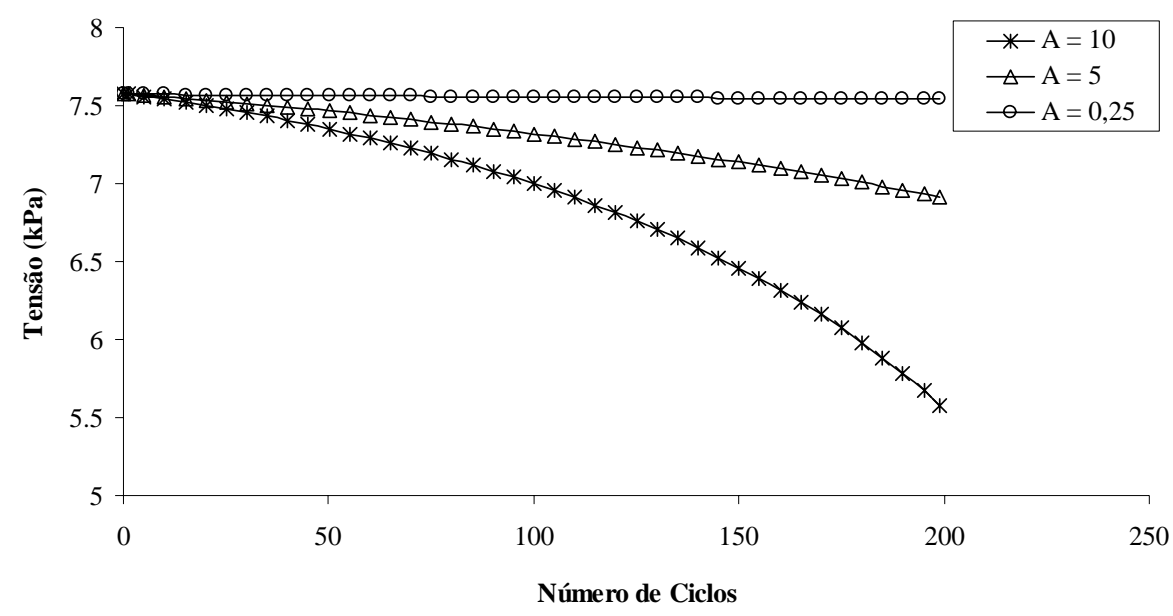

Figura 8. Tensões horizontais no fundo da camada asfáltica

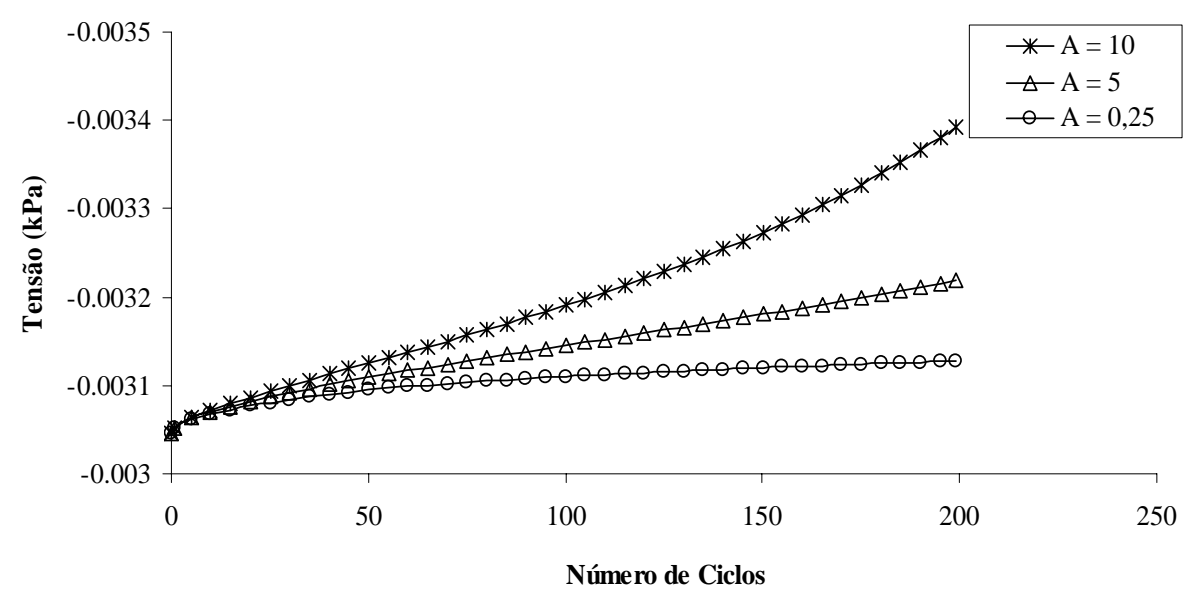

Figura 9. Tensões verticais no topo da base

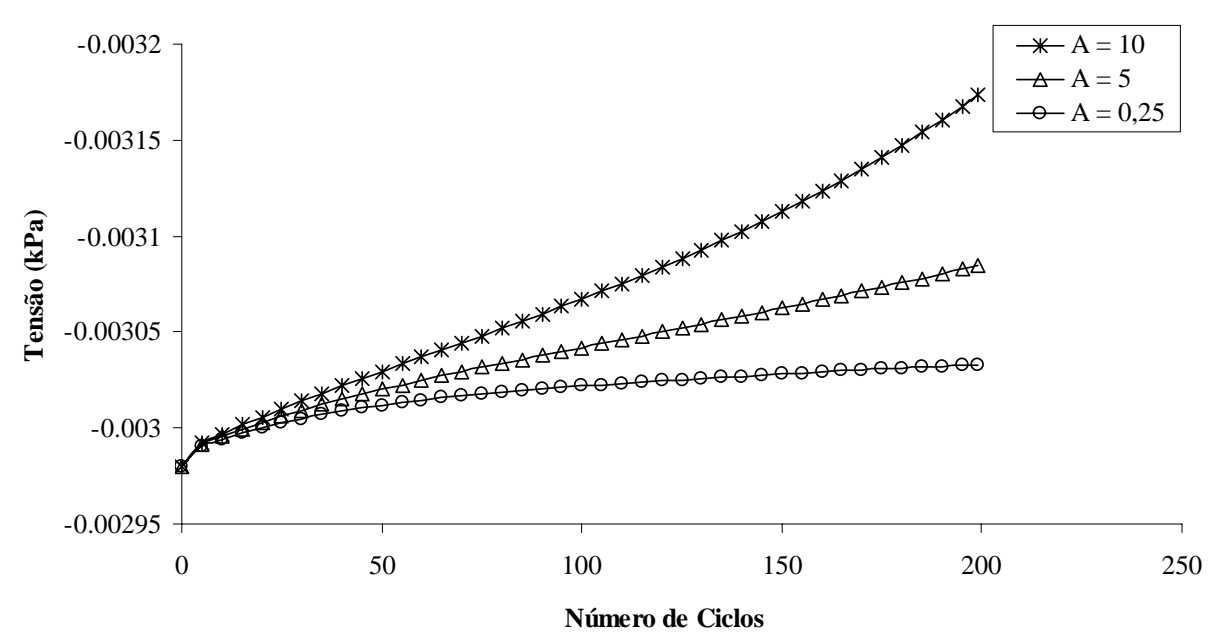

Figura 10. Tensões verticais no topo do subleito 


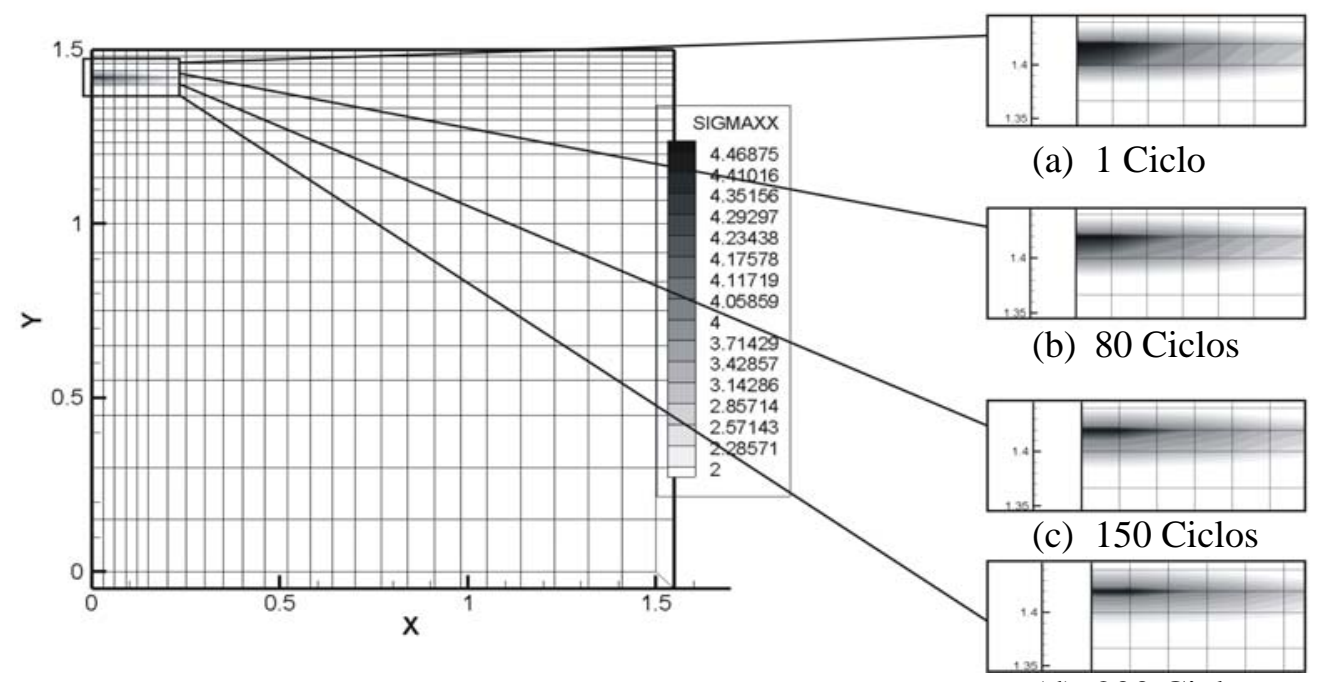

(d) 200 Ciclos

Figura 11. Redução das tensões horizontais no fundo do revestimento com o aumento do número de ciclos de carga

gerado pelos carregamentos. $\mathrm{O}$ trabalho apresenta também a formulação do problema de valor de contorno inicial, assim como mostra algumas simulações de problemas de pavimentos. Fatores ambientais, como variação de temperatura, não foram considerados no modelo neste momento. Os resultados indicam principalmente que o modelo pode levar em consideração os fenômenos de fadiga e deformação permanente nos pavimentos, dois dos principais fatores de deteriorização dos mesmos. Também é apresentado como as tensões na camada asfáltica (comportamento viscoelástico com dano) decrescem à medida que o dano aumenta. Por outro lado, com a redução da capacidade de absorção das tensões pelo revestimento, as cargas são redistribuídas nas camadas inferiores, que conseqüentemente passam a ser mais exigidas. Também foram apresentados diferentes valores para os parâmetros de dano, mostrando a influência que estes têm na deterioração dos pavimentos. O modelo apresentado não levou em consideração o dano para as camadas granulares, para simplificar a análise. Futuros estudos pretendem estender a capacidade do modelo para simular a evolução do dano em camadas granulares, mas fica desde já claro o potencial da abordagem utilizada em contribuir para uma melhor compreensão do comportamento estrutural dos pavimentos asfálticos e, conseqüentemente, melhor dimensionamento dos mesmos. A abordagem traz a vantagem de apresentar uma forma de se quantificar o dano em revestimentos asfálticos. Faz-se isso com parâmetros numéricos específicos, tornando possível a definição objetiva de um fenômeno freqüentemente tratado de forma abstrata (dano em misturas), e contribuindo para uma melhor compreensão e definição do mesmo, que é um dos desafios da Mecânica dos Pavimentos.

\section{REFERÊNCIAS BIBLIOGRÁFICAS}

Allen, D. H.; Searcy, C. R. (2001) A micromechanical model for a viscoelastic cohesive zone. International Journal of Fracture, v. 107, p. 159-176.

Boussinesq, J. (1885) Application des potentiels a l'étude de l'equilibre et du mouvement des solids elastiques, Gauthier-Villars, Paris.

Christensen, R. M. (1982) Theory of Viscoelasticity (2a ed.). Academic, New York.

Freitas, F.A.C. (2007) A theoretical and experimental technique to measure fracture properties in viscoelastic solids. $\mathrm{PhD}$ dissertation, $\mathrm{U}-$ niversity of Nebraska-Lincoln.

Gibson, N.H.; C.W.; Schartz; R.A.; Schapery; Witczack M.W. (2002) Viscoelastic, Viscoplastic, and Damage Modeling of Asphalt Concrete in Unconfined Compression. TRB 2003 Annual Meeting CDROM.

Huang, Y.H. (2004) Pavement Analysis and Design. $2^{\mathrm{a}}$ ed. Prentice Hall, Englewoods Cliffs, New Jersey, USA.

Kim, Y.R.; D.H. Allen; Seidel, G.D. (2006) Damage-induced modeling of elastic-viscoelastic randomly oriented particulate composites. Journal of Engineering Materials and Technology, v. 128, p. 1827.

Lee, H.J.; Kim, Y.R. (1998) Viscoelastic constitutive model for asphalt concrete under cyclic loading. Journal of Engineering Mechanics, v. 124 , no 1 , p. 32-40.

Lee, H.J.; Kim, Y.R. (1998) Viscoelastic continuum damage model of asphalt concrete with healing. Journal of Engineering Mechanics, v. 124, n. 11, p. 1224-1232.

Lutif, J.E.S. (2007) The use of fundamental properties of mixture constituents to evaluate moisture susceptibility of asphalt concrete mixtures. Tese de Mestrado. University of Nebraska-Lincoln, Lincoln, Nebraska, Estados Unidos.

Medina, J.; Motta, L.M.G. (2005) Mecânica dos Pavimentos. 2a Edição, Rio de Janeiro.

MTOOL (2001) Bidimensional Mesh Tool, Versão 4.0. Grupo de Tecnologia em Computação Gráfica,TeCGraf5, PUC-Rio, 1997.

NCHRP (2004) Guide for mechanistic-empirical design of new and rehabilitated pavement structures. National Cooperative Highway Research Program, 1-37A Research Report.

Park, S. W.; Kim, Y.R.; Schapery, R.A. (1996) A viscoelastic continuum damage model and its application to uniaxial behavior of asphalt concrete. Mechanics of Materials, v. 24, p. 241-255.

Schapery, R.A.; Tielking, J.T. (1977) Investigation of tire-pavement interaction during maneuvering, v. 1 - Theory and results. Report No. FHWA-RD-78-72, Federal Highway Administration, Washington, D.C., Estados Unidos.

Schapery, R.A. (1984) Correspondence principles and a generalized jintegral for large deformation and fracture analysis of viscoelastic media. International Journal of Fracture. v. 25, p. 195-223. 
Schapery, R.A. (1990) A theory of mechanical behavior of elastic media with growing damage and other changes in structure. J. Mech. Phys. Solids, v. 38, p. 215-253.

Schapery, R.A. (1990) Simplifications in the behavior of viscoelastic composites with growing damage. Symposium on Inelastic Deformation of Composite Materials, Troy, New York. Springer, New York-Wien, p. 193-214.

Seidel, G.D.; D.H. Allen; K.L.E. Helms; Groves, S.E. (2004) A model for predicting the evolution of damage in viscoelastic particlereinforced composites. Mechanics of Materials.

Souza, F.V.; Soares, J.B. (2003) Efeito da consideração do comportamento viscoelástico linear do revestimento no cálculo de tensões e deslocamentos em pavimentos asfálticos. XVII Congresso de Pesquisa e Ensino em Transportes, ANPET, Brasil.

Souza, F.V. (2005) Modelo multi-escala para análise estrutural de compósitos viscoelásticos susceptíveis ao dano. Tese de Mestrado. Universidade Federal do Ceará, Fortaleza, Ceará, Brasil.

Tekscan Incorporation. Disponível em: <www.tekscan.com>. Acesso em 2007.

Tielking, J.T. (1983) A finite element tire model. Tire Science and Techno$\log y, 11(1-4)$, p. 50-63.

Tielking, J.T.; Roberts, F.L. (1987) Tire contact pressure and its effect on pavement strain. Journal of Transportation Engineering, v. 113, No. 1, ASCE.

Zocher, M.A. (1995) A thermoviscoelastic finite element formulation for the analysis of composites. PhD dissertation, Texas A\&M University, College Station, EUA. 